\title{
PRODUCCIÓN DE SEMILLAS EN YACÓN (Smallanthus sonchifolius (Poepp. \& Endl.)) MEDIANTE TÉCNICAS DE POLINIZACIÓN CONTROLADAS
}

\section{YACON (Smallanthus sonchifolius (Poepp. \& Endl.)) SEED PRODUCTION THROUGH CONTROLLED POLLINATION TECHNIQUES}

\author{
Iván Manrique ${ }^{1}$, Roberto Gonzales ${ }^{1}$, Andrés Valladolid ${ }^{2}$, Raúl Blas ${ }^{3}$ y Luis Lizárraga ${ }^{4}$
}

\begin{abstract}
Resumen
El yacón, una raíz de origen andino, es una fuente abundante de fructooligosacáridos (FOS) y compuestos fenólicos. Su consumo ha sido asociado con la prevención de enfermedades crónicas (dislipidemias y resistencia a la insulina), cáncer de colon, estreñimiento, entre otras propiedades. La propagación del yacón es eminentemente clonal. Se han sugerido mecanismos de androesterilidad y una escasa o nula viabilidad en las semillas, lo que imposibilitarían su reproducción sexual en condiciones naturales y controladas. En el presente trabajo se realizaron cruzamientos entre seis accesiones de yacón (cuatro octoploides y dos dodecaploides) con la finalidad de obtener híbridos F1 y generar información acerca de la producción y viabilidad de las semillas. Para ello, se usó un diseño de cruzas dialélicas, directas y recíprocas, de las que se generaron 30 cruzas F1. En total se polinizaron 1746 inflorescencias y se obtuvieron 1181 semillas llenas. En todos los cruzamientos planificados se produjo semilla, excepto en aquellos cruzamientos en los que los progenitores dodecaploides actuaron como femeninos. Cuando las semillas llenas fueron escarificadas y sembradas en sustrato orgánico se logró obtener un porcentaje de germinación de $38.4 \%$. En promedio se necesitaron polinizar cuatro inflorescencias de yacón por cada semilla viable que se obtuvo. La factibilidad de producir semillas de yacón empleando polinizaciones controladas abre oportunidades para plantear investigaciones en la biología reproductiva del cultivo, probar la teoría del origen alopoliploide del yacón, obtener variedades mejoradas y estudiar nuevas estrategias de conservación y distribución de germoplasma de yacón.
\end{abstract}

Palabras clave: Híbrido, yacón, semilla, polinización, octoploide, dodecaploide

\begin{abstract}
Yacon, an Andean root crop, is a plentiful source of fructooligosaccharides (FOS) and phenolic compounds. Its consumption has been related to the prevention of chronic diseases (dyslipidemia and insulin resistance), colon cancer, constipation, among other properties. Yacon reproduction is eminently clonal. Mechanisms of male sterility and scarce or null viability in the seeds have been suggested in yacon, which would preclude its sexual reproduction in natural or controlled conditions. In this work, six yacon accessions (four octoploids and two dodecaploids) were cross-pollinated in order to obtain F1 hybrids and generate information about the production and viability of the seeds. A dialelic design was used, with direct and reciprocal crosses, which generated $30 \mathrm{~F} 1$ crosses. A total of 1746 inflorescences were pollinated and 1181 seeds were obtained. All crosses produced seeds, except those crosses where dodecaploid progenitors worked as female. A high value of germination (38.39\%) was obtained when the seeds were scarified and planted in an organic substrate (moss and peat moss). On average it was necessary to pollinate four inflorescences to obtain one viable yacon seed. The feasibility to produce yacon seeds opens opportunities to propose studies related to the reproductive biology, test the allopolyploid origin of the yacon, obtain breeding materials and to study new strategies for the conservation and distribution of yacon germplasm.
\end{abstract}

Key words: Hybrid, yacon, seed, pollination, octoploid, dodecaploid

\section{Introducción.}

El yacón (Smallanthus sonchifolius) es una raíz originaria de la región Andina con enormes posibilidades de desarrollo para el mercado de alimentos funcionales (Seminario et al., 2003). Su consumo reconstituye la microflora benéfica del colon, reduce el colesterol sanguíneo, mejora la asimilación de calcio, corrige el estreñimiento y 
fortalece el sistema inmunológico (Geyer et al., 2008; Genta et al., 2009). El yacón además es un alimento ideal para diabéticos y para personas que desean bajar de peso ya que su consumo no eleva la concentración de glucosa en la sangre y aporta muy pocas calorías a la dieta. Estas propiedades están fuertemente asociadas a los compuestos fenólicos y a los fructooligosacáridos (FOS), los cuales pueden variar su contenido en las raíces dependiendo de la variedad, el ambiente y su interacción (Manrique, comunicación personal). Se han reportado diferencias significativas para el contenido de FOS y compuestos fenólicos en diferentes accesiones de yacón sembradas en la República Checa (Valentová et al., 2006) y en Ecuador (Hermann et al., 1999). De igual modo se han reportado diferencias muy grandes para el rendimiento de raíces que varían entre 16 y 100 t/ha (Seminario et al., 2003).

La colección de germoplasma de yacón del Instituto Nacional de Innovación Agraria (INIA) está constituida por 326 accesiones colectadas de diferentes localidades del Perú (Soto, 2012). En esta colección se han encontrado materiales promisorios con atributos de valor agronómico, nutracéutico y nutricional muy variables, que podrían ser usados en programas de mejoramiento genético para desarrollar nuevas variedades, y eventualmente reemplazar a las variedades comerciales que actualmente son usadas por la agroindustria. Sin embargo, existen dificultades para obtener semilla sexual de yacón debido a razones que aún son poco comprendidas.

En condiciones naturales o controladas el yacón produce muy pocas semillas, la mayor parte de las cuales son vanas o no alcanzan un desarrollo adecuado dentro del aquenio (Seminario et al., 2003). Mansilla et al. (2010) han reportado que tan solo el $11.2 \%$ de los aquenios que se produjeron en un campo de polinización abierta en Huánuco contenían semillas, las cuales a su vez alcanzaron una viabilidad que apenas llegó a 0.25\%. Meza (1995) obtuvo también valores muy bajos de viabilidad; de 300 semillas cosechadas en Cusco solo una germinó. En Japón, Sugiura et al. (2007) lograron obtener una sola semilla viable después de polinizar 382 capítulos; el híbrido dio origen a una variedad nueva de yacón conocida con el nombre de Sarada Otome. Contrario a los resultados anteriores, Chicata (1998) obtuvo una viabilidad alta (29.1\%) en las semillas que fueron colectadas a partir de 67 accesiones que crecieron en Cusco (357 de 1227 semillas lograron germinar y producir híbridos). Sin embargo, la viabilidad resultó mucho mayor (64.9\%) en las semillas llenas (316 de 487 semillas llenas lograron germinar).

La baja tasa de formación de semilla llena y viable ha sido discutida por diferentes autores.
Aparentemente no existen problemas de receptividad en el estigma. Mansilla et al. (2010) usando la técnica del burbujeo de estigmas y estilos en peróxido de hidrógeno (Dafni, 1992) obtuvieron valores entre 76 y 94\% para la receptividad del estigma. La baja fertilidad del yacón ha sido asociada al grano de polen por Grau \& Slanis (1996; citados por Grau \& Rea 1997), quienes reportaron una fertilidad que varió entre $0 \quad$ y $30 \%$ para condiciones de Argentina. Mansilla et al. (2010) han reportado valores muy bajos de germinación in vitro para granos de polen $(0.16 \%)$ en plantas que crecieron en Huánuco. Sin embargo, Soto (1998) utilizando la técnica de tinción con aceto carmín, encontró más del 55\% de viabilidad en granos de polen obtenidos de plantas crecidas en Cajamarca.

Según Ishiki et al. (1997) el yacón tiene un origen alopoliploide, originado por cruzamientos complejos entre S. macroscyphus $(2 n=28, A=7)$ y $S$. riparius $(2 n=32, B=8)$. Este origen explicaría la naturaleza octoploide $(2 \mathrm{n}=6 \mathrm{~A}+2 \mathrm{~B}=58) \quad \mathrm{y}$ dodecaploide $(2 n=9 A+3 B=87)$ en aquellas accesiones que han sido caracterizadas citológicamente por Salgado (1996), Araujo (1998) y Frías de Fernández et al. (2000). El origen híbrido del yacón y su naturaleza alopoliploide podría ser también una de las causas de la escasa producción de semillas y de su baja viabilidad.

Los objetivos del presente trabajo de investigación fueron: 1) Evaluar la viabilidad y la germinación de semillas obtenidas a partir de cruzamientos dirigidos entre seis accesiones de yacón con diferentes atributos agronómicos, nutracéuticos y nutricionales. 2) Obtener híbridos F1 viables de yacón.

\section{Materiales y métodos.}

Material vegetal.

Seis accesiones de yacón del banco de germoplasma del INIA fueron seleccionadas para realizar los cruzamientos. Las accesiones fueron seleccionadas por sus atributos agronómicos, nutricionales y nutracéuticos (Tabla 1 y Figura 1). Como material de siembra se usaron propágulos de aproximadamente $50 \mathrm{~g}$ de peso. Por cada accesión de yacón se instalaron cuatro parcelas, cada una conteniendo 60 plantas. Se usó un distanciamiento de $0.8 \mathrm{~m}$ entre plantas y $1.00 \mathrm{~m}$ entre surcos. La siembra se hizo en el mes de junio de 2011 en la comunidad de Rosapata (10¹1'09” LS, 7609'13” LO), ubicada sobre los $2792 \mathrm{msnm}$ en el distrito de Ambo (provincia de Ambo, departamento de Huánuco). Una réplica de este ensayo, en condiciones de invernadero, fue instalado el mes de setiembre de 2011 en el Centro Agronómico K’ayra (133’24” LS, 7152’30” LO), ubicado sobre los 3219 msnm en el distrito San Jerónimo (provincia Cusco, departamento Cusco). 
Tabla 1. Datos pasaporte y principales características de las seis accesiones de yacón seleccionadas del banco de germoplasma del INIA para la realización de los cruzamientos.

\begin{tabular}{|c|c|c|c|c|c|c|}
\hline $\begin{array}{l}\text { Nombre de } \\
\text { accesión }\end{array}$ & Código & Departamento & Provincia & Distrito & $\begin{array}{l}\text { Altitud } \\
\text { (msnm) }\end{array}$ & $\begin{array}{c}\text { Características de } \\
\text { las accesiones }\end{array}$ \\
\hline UNALM 2 & V4 & Huánuco & & & & $\begin{array}{l}\text { Alta productividad } \\
\text { de raíces, alto Brix } \\
\text { materia seca y } \\
\text { FOS. }\end{array}$ \\
\hline ZSY044 & V11 & Puno & Carabaya & $\begin{array}{l}\text { San } \\
\text { Gabán }\end{array}$ & 1200 & $\begin{array}{c}\text { Tiene antocianinas } \\
\text { y alta actividad } \\
\text { antioxidante }\end{array}$ \\
\hline PER007663 & V18 & Ayacucho & Huanta & Sivia & 1820 & $\begin{array}{c}\text { Resistente al } \\
\text { pardeamiento }\end{array}$ \\
\hline AMM5163 & V24 & Ancash & Carhuaz & Shilla & 3400 & $\begin{array}{l}\text { Alto Brix, materia } \\
\text { seca, FOS y } \\
\text { compuestos } \\
\text { fenólicos. }\end{array}$ \\
\hline UNALM4 & V28 & Huánuco & & & & $\begin{array}{c}\text { Alta productividad } \\
\text { de raíces y alto } \\
\text { FOS }\end{array}$ \\
\hline Testigo & $\mathrm{T}$ & & & & & Variedad Control \\
\hline
\end{tabular}

horas a una temperatura de $4^{\circ} \mathrm{C}$. Inmediatamente después las raíces se fijaron en una solución de Carnoy (3 ml de etanol absoluto por $1 \mathrm{ml}$ de ácido acético glacial) y se almacenaron a $4^{\circ} \mathrm{C}$ por 20 horas. Finalmente las raíces fueron maceradas en $\mathrm{HCl} 1 \mathrm{~N}$ a $60^{\circ} \mathrm{C}$ por 9 minutos y luego teñidas con aceto-orceina al $2 \%$ por 24 horas a temperatura ambiente. El conteo de cromosomas se realizó con un microscopio óptico (15x100). Se examinaron al menos 10 células metafásicas para realizar el conteo de cromosomas y se usó la moda para establecer la ploidía de las accesiones.

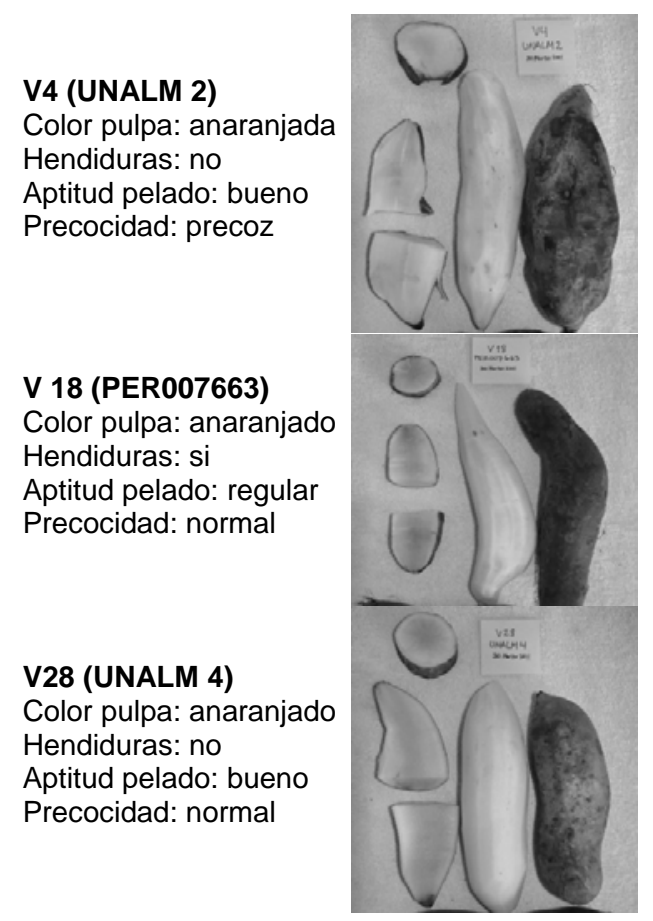

V11 (ZS Y 044)

Color pulpa: blanco con pigmentos morados Hendiduras: si Aptitud pelado: regular Precocidad: normal

\section{24 (AMM5163)}

Color pulpa: anaranjado Hendiduras: si Aptitud pelado: regular Precocidad: precoz

\section{Testigo}

Color pulpa: anaranjado Hendiduras: poco Aptitud pelado: regular Precocidad: normal

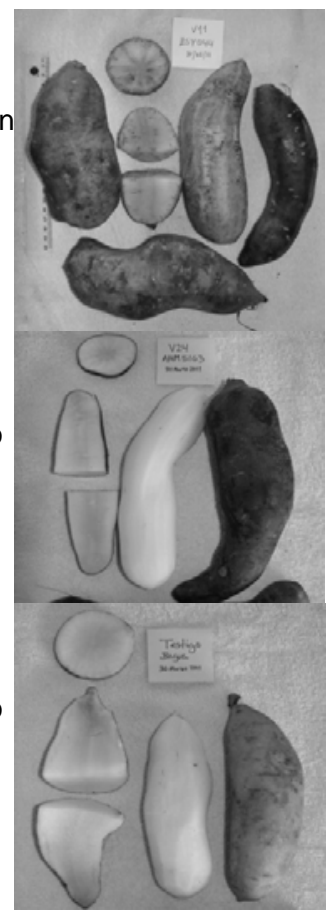

Figura 1. Precocidad y otras características de interés en las seis accesiones de yacón utilizadas como progenitores.

Ploidía y número de cromosomas.

La determinación del número cromosómico de las seis accesiones de yacón se realizó con la técnica del squash o aplastado a partir de células metafásicas obtenidas de puntas de raíces de plantas jóvenes desarrolladas en condiciones de invernadero. Se colectaron puntas de raíces jóvenes entre las 7:40 y 8:10 am en un prefijador (8-hidroxiquinolina 0.002 M). Las raíces fueron expuestas al prefijador por 3
Polinizaciones.

Se empleó un diseño de cruzas dialélicas, directas y recíprocas, de las que se generaron 30 cruzas F1 (Tabla 2). Las polinizaciones en Ambo se efectuaron en campo abierto, en tres oportunidades: la última semana de febrero, la tercera semana de marzo y la última semana de abril de 2012. Las polinizaciones en Cusco se realizaron en condiciones de invernadero, en tres oportunidades: la segunda y cuarta semana de 

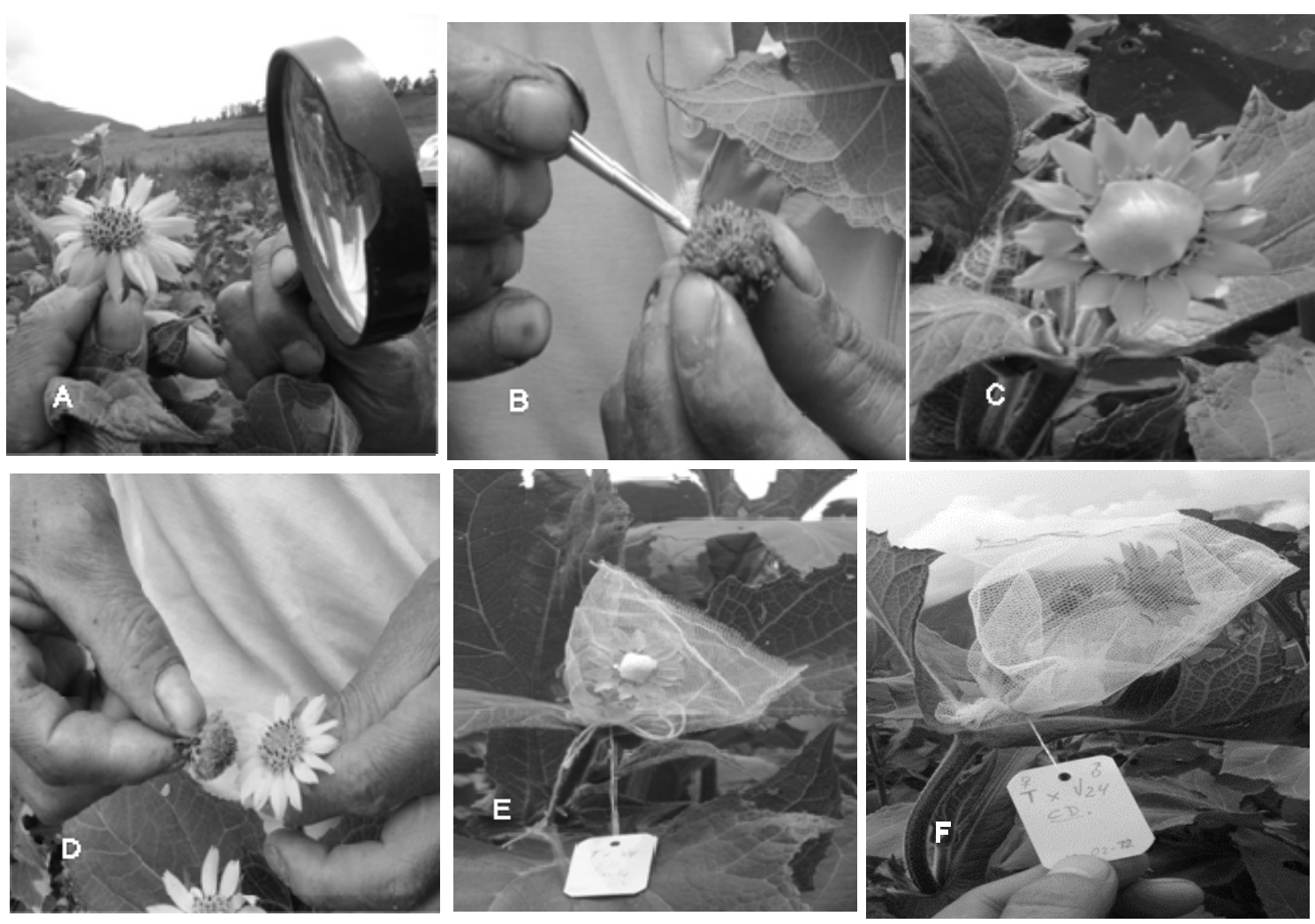

Figura 2. Método de polinización. Paso 1 (A y B). Preparación de capítulos donantes de granos de polen. Con la ayuda de una luna de aumento se seleccionan capítulos que tengan al menos $60 \%$ de flores tubulares en antesis (dehiscentes). Con una pinza se retiran las flores liguladas (femeninas) y el involucro, conservando intacto el disco, las flores tubulares y el pedúnculo. Luego se aplastan con cuidado las flores tubulares para forzar la dehiscencia y con ello el aumento de granos de polen. El disco queda listo para ser usado a modo de una pequeña brocha para realizar las polinizaciones. Paso 2 (C). Preparación de plantas y sus capítulos para ser usadas como progenitores femeninos. Se seleccionan plantas que servirán de progenitores femeninos o receptoras de polen. Los capítulos de estas plantas deben ser de preferencia grandes, con las flores liguladas bien expandidas. Se debe constatar además que las ramitas del estigma bífido de las flores liguladas estén bien separadas, lo cual es un indicador de que el estigma está receptivo. Finalmente se cubren las flores tubulares con cinta adhesiva para evitar la contaminación de polen extraño y autopolinización. Paso 3 (D, E y F). Polinización de los capítulos en las plantas seleccionadas como progenitores femeninos. Usar los discos en forma de brochitas y llenos de polen (ver paso 1) para impregnar con polen los estigmas de las flores femeninas previamente preparadas en el Paso 2. Ya que el polen de yacón es muy pegajoso es necesario pasar dos o tres veces el disco sobre los estigmas. Luego se verifica con una luna de aumento la presencia de granos de polen en el estigma. A continuación se cubren los capítulos polinizados con una bolsa de malla fina y se etiquetan identificando los progenitores, la fecha y el tipo de cruza. Finalmente se eliminan de la planta los capítulos que no fueron polinizados y se realiza una aplicación foliar de Calcio, Boro y Zinc (100ml/20 l) para favorecer el cuajado y llenado de los frutos.

mayo, y la segunda semana de junio de 2012. Los capítulos polinizados fueron cosechados cuando las inflorescencias estuvieron completamente secas, aproximadamente un mes después de finalizadas las últimas polinizaciones. Las semillas colectadas se almacenaron en un lugar seco y aireado hasta el momento de hacer las pruebas de germinación. El método empleado en los cruzamientos es detallado en la Figura 2. Un total de 1746 capítulos fueron polinizados en Ambo para los 30 tipos de cruzas, incluyendo las cruzas directas y las recíprocas; mientras que en Cusco fueron polinizados 300 capítulos (Tabla 2).

Viabilidad y germinación de semillas.

Los aquenios de las inflorescencias polinizadas fueron procesados para identificar los frutos que contenían semillas llenas, parcialmente llenas y vanas. Las semillas vanas se identificaron fácilmente debido 

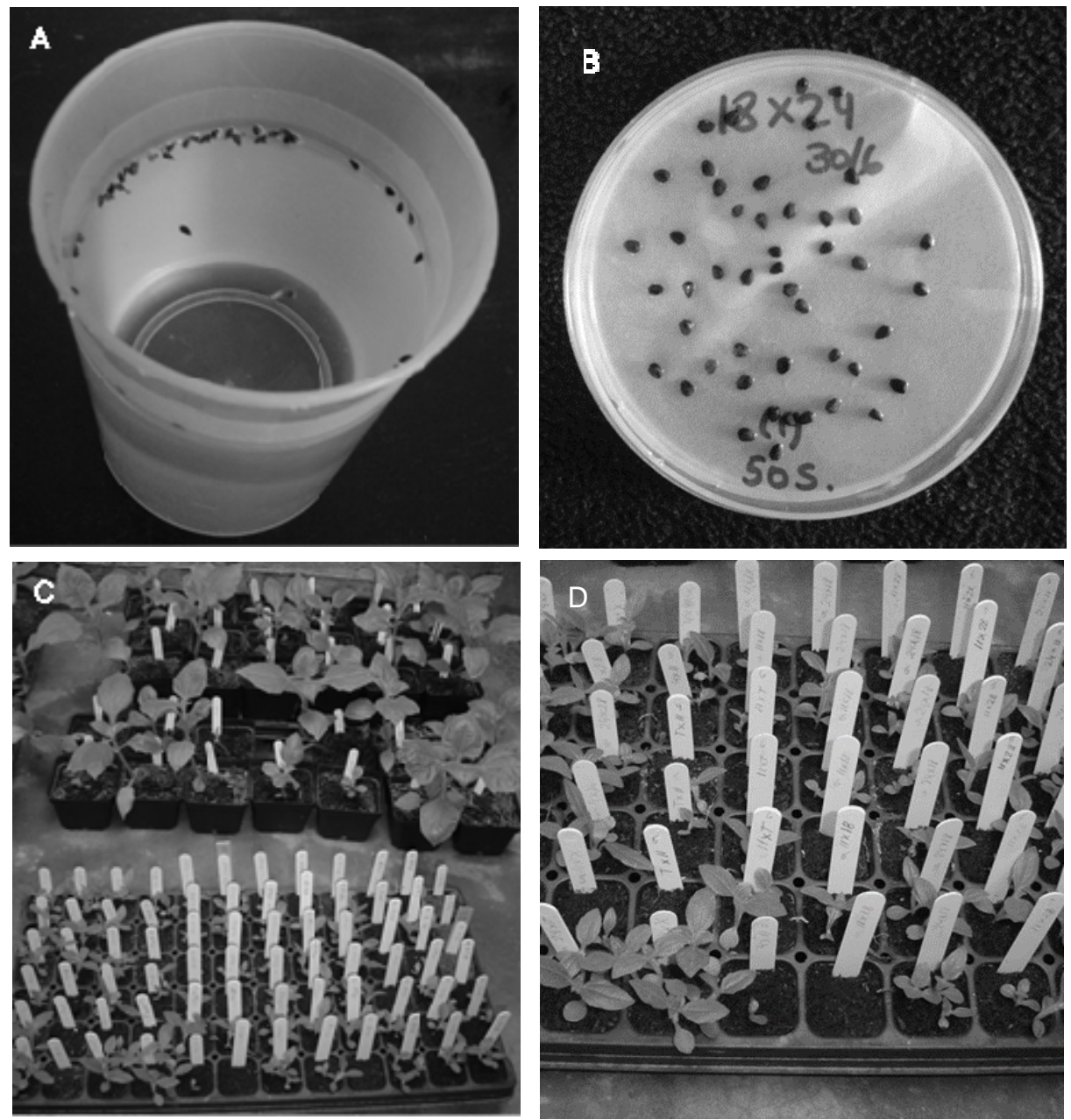

Figura 3. Germinación de semillas de yacón. A: Las semillas que flotaron sobre un vaso conteniendo agua fueron consideradas vanas o vacías. B: Prueba de germinación en placas Petri conteniendo papel filtro humedecido con agua estéril. C y D: Prueba de germinación usando bandejas germinadoras conteniendo sustrato orgánico (turba y musgo).

a que se desintegraron fácilmente cuando se aplicó una presión mecánica leve con los dedos de la mano. Las semillas parcialmente llenas se reconocieron porque flotaron sobre la superficie de un recipiente conteniendo agua (Figura 3). Las semillas que permanecieron sumergidas en el fondo del recipiente fueron consideradas llenas y fueron las únicas que se emplearon para realizar los ensayos de germinación.

Para las pruebas de germinación se probaron dos métodos (Figura 3). El primer método consistió en la incubación directa de las semillas en placas Petri conteniendo papel Whatman humedecido con agua destilada estéril. Las semillas se remojaron previamente por 12 horas en agua destilada estéril para ablandar el pericarpio. Se usaron 50 semillas por placa Petri y se hicieron evaluaciones diarias durante seis semanas para constatar la emergencia de la radícula en las semillas. El segundo método consistió en la siembra de las semillas en bandejas germinadoras conteniendo sustrato orgánico (turba y musgo). Antes de la siembra en el sustrato orgánico las semillas fueron escarificadas con un abrasivo leve (papel lejía) hasta lograr un pequeño orificio en el pericarpio. Las semillas fueron sembradas a $1 \mathrm{~cm}$ de profundidad en el sustrato.

Las pruebas de germinación en placas Petri se realizaron en el mes de julio de 2012 y las pruebas de germinación en sustrato orgánico en octubre de 2012. El porcentaje de viabilidad de las semillas fue calculado a partir del cociente obtenido al dividir el número total de semillas llenas que lograron germinar y el número total de semillas llenas que se obtuvieron a partir de las polinizaciones. Las semillas que 
Tabla 2. Número de inflorescencias (polinizadas y cosechadas) y número de semillas llenas obtenidas de los cruzamientos directos (CD) y recíprocos (CR) efectuados en Rosapata (Ambo) y Cusco.

\begin{tabular}{|c|c|c|c|c|c|c|c|c|}
\hline $\begin{array}{l}\text { Progenitor } \\
\text { Femenino }\end{array}$ & $\begin{array}{l}\text { Progenitor } \\
\text { Masculino }\end{array}$ & $\begin{array}{c}\text { Tipo de } \\
\text { cruza }\end{array}$ & $\begin{array}{l}\text { Total capítulos } \\
\text { polinizados } \\
\text { (Ambo) }\end{array}$ & $\begin{array}{c}\text { Total } \\
\text { capítulos } \\
\text { cosechados } \\
\text { (Ambo) }\end{array}$ & $\begin{array}{c}\text { Número } \\
\text { semillas } \\
\text { llenas } \\
\text { (Ambo) }\end{array}$ & $\begin{array}{c}\text { Total capítulos } \\
\text { polinizados } \\
\text { (Cusco) }\end{array}$ & $\begin{array}{c}\text { Total } \\
\text { capítulos } \\
\text { cosechados } \\
\text { (Cusco) }\end{array}$ & $\begin{array}{c}\text { Número } \\
\text { semillas } \\
\text { llenas } \\
\text { (Cusco) }\end{array}$ \\
\hline V4 & V11 & CD & 57 & 52 & 50 & 10 & 0 & 0 \\
\hline V4 & V18 & CD & 57 & 52 & 41 & 10 & 0 & 0 \\
\hline V4 & V24 & CD & 97 & 90 & 25 & 10 & 0 & 0 \\
\hline V4 & V28 & CD & 57 & 31 & 20 & 10 & 0 & 0 \\
\hline V4 & $\mathrm{T}$ & CR & 57 & 47 & 22 & 10 & 0 & 0 \\
\hline V11 & V4 & CR & 57 & 50 & 84 & 10 & 0 & 0 \\
\hline V11 & V18 & CD & 57 & 52 & 37 & 10 & 0 & 0 \\
\hline V11 & V24 & CD & 57 & 54 & 38 & 10 & 16 & 0 \\
\hline V11 & V28 & CD & 57 & 49 & 46 & 10 & 2 & 0 \\
\hline V11 & $\mathrm{T}$ & CR & 57 & 47 & 23 & 10 & 2 & 0 \\
\hline V18 & V4 & CR & 87 & 79 & 244 & 10 & 0 & 0 \\
\hline V18 & V11 & CR & 57 & 41 & 68 & 10 & 0 & 0 \\
\hline V18 & V24 & CD & 53 & 44 & 99 & 10 & 0 & 0 \\
\hline V18 & V28 & CD & 57 & 50 & 92 & 10 & 0 & 0 \\
\hline V18 & $\mathrm{T}$ & CR & 64 & 47 & 117 & 10 & 0 & 0 \\
\hline V24 & V4 & CR & 64 & 58 & 10 & 10 & 0 & 0 \\
\hline V24 & V11 & CR & 57 & 53 & 32 & 10 & 5 & 0 \\
\hline V24 & V18 & CR & 74 & 72 & 42 & 10 & 0 & 0 \\
\hline V24 & V28 & CD & 57 & 43 & 17 & 10 & 0 & 0 \\
\hline V24 & $\mathrm{T}$ & CR & 57 & 58 & 15 & 10 & 0 & 0 \\
\hline V28 & V4 & CR & 57 & 50 & 0 & 10 & 0 & 0 \\
\hline V28 & V11 & CR & 65 & 57 & 0 & 10 & 16 & 0 \\
\hline V28 & V18 & CR & 23 & 14 & 0 & 10 & 0 & 0 \\
\hline V28 & V24 & CR & 57 & 55 & 0 & 10 & 0 & 0 \\
\hline V28 & $\mathrm{T}$ & CR & 57 & 49 & 1 & 10 & 5 & 0 \\
\hline $\mathrm{T}$ & V4 & CD & 57 & 18 & 0 & 10 & 0 & 0 \\
\hline $\mathrm{T}$ & V11 & CD & 57 & 37 & 20 & 10 & 4 & 0 \\
\hline $\mathrm{T}$ & V18 & CD & 22 & 19 & 1 & 10 & 0 & 0 \\
\hline $\mathrm{T}$ & V24 & CD & 57 & 10 & 0 & 10 & 0 & 0 \\
\hline $\mathrm{T}$ & V28 & CD & 57 & 22 & 0 & 10 & 0 & 0 \\
\hline Total & & & 1746 & $1400^{*}$ & $1144^{* *}$ & 300 & 50 & 0 \\
\hline
\end{tabular}

*Veinte capítulos adicionales fueron cosechados, en los cuales se perdió la identificación del progenitor masculino. Ocho capítulos se identificaron como V4xBulk y los otros doce capítulos como V24xBulk.

** De los capítulos V4xBulk se obtuvieron 20 semillas llenas y de los capítulos V24xBulk se obtuvieron 17 semillas llenas.

lograron germinar se llevaron a campo definitivo cuando alcanzaron una altura aproximada de $20 \mathrm{~cm}$.
Resultados.

Ploidía y número de cromosomas.

La Tabla 3 muestra los resultados del número de cromosomas y la ploidía de las seis accesiones de yacón usadas como progenitores. Cuatro accesiones 
(V4, V11, V18 y V24) resultaron octoploides $(2 n=58)$ y dos accesiones (V28 y el Testigo) resultaron dodecaploides $(2 n=87)$. El número de cromosomas y la ploidía de las seis accesiones guardan correspondencia con lo reportado previamente por otros autores (Salgado, 1996; Araujo, 1998; Frías de Fernández et al., 2000), quienes encontraron también materiales octoploides y dodecaploides de yacón. La prevalencia de las accesiones octoploides resultó claramente superior a las accesiones dodecaploides, lo cual confirma los resultados reportados por Hermann et al. (1999) y Salgado (1996). La Figura 4 muestra los
Tabla 3. Resultados del número de cromosomas y nivel de ploidía en las seis accesiones de yacón empleadas en las polinizaciones

\begin{tabular}{lcl}
\hline Accesión & $\begin{array}{c}\text { Número de } \\
\text { cromosomas }\end{array}$ & \multicolumn{1}{c}{ Ploidía } \\
\hline UNALM2 (V4) & 58 & Octoploide $(2 n=58)$ \\
ZYS044 (V11) & 58 & Octoploide $(2 n=58)$ \\
PER007663 (V18) & 58 & Octoploide $(2 n=58)$ \\
AMM5163 (V24) & 58 & Octoploide $(2 n=58)$ \\
UNALM4 (V28) & 87 & Dodecaploide $(2 n=87)$ \\
Testigo (T) & 87 & Dodecaploide $(2 n=87)$ \\
\hline
\end{tabular}

de cruzamiento en Ambo y en

Tabla 4. Resultados de la prueba de germinación utilizando el método de placas Petri conteniendo papel filtro humedecido con agua estéril.

\begin{tabular}{cccccc}
\hline Progenitor & Progenitor & $\begin{array}{c}\text { Nro. } \\
\text { Femenino }\end{array}$ & Masculino & Nro. semillas \\
procesados & llenas & $\begin{array}{c}\text { Nro. semillas } \\
\text { germinadas y } \\
\text { trasplantadas a } \\
\text { campo }\end{array}$ & $\begin{array}{c}\text { Porcentaje de } \\
\text { germinación }\end{array}$ \\
\hline V4 & V11 & 12 & 31 & 0 & $0 \%$ \\
V4 & V18 & 52 & 41 & 0 & $0 \%$ \\
V4 & V24 & 89 & 25 & 0 & $0 \%$ \\
V4 & V28 & 21 & 11 & 0 & $0 \%$ \\
V4 & T & 47 & 22 & 0 & $0 \%$ \\
V11 & V4 & 2 & 3 & 0 & $0 \%$ \\
V11 & V28 & 2 & 1 & 0 & $0 \%$ \\
V18 & V4 & 76 & 235 & 0 & $0 \%$ \\
V18 & V11 & 41 & 68 & 0 & $0 \%$ \\
V18 & V24 & 43 & 99 & 0 & $0 \%$ \\
V18 & V28 & 49 & 92 & 0 & $0 \%$ \\
V18 & T & 47 & 117 & 0 & $0 \%$ \\
V24 & V4 & 2 & 1 & 0 & $0 \%$ \\
V24 & V18 & 1 & 2 & 0 & $0 \%$ \\
T & V11 & 23 & 1 & 0 & $0 \%$ \\
T & V18 & 19 & 1 & 0 & $0 \%$ \\
V4 & Bulk & 4 & 9 & 0 & $0 \%$ \\
Total & & 530 & 759 & 0 & $0 \%$ \\
\hline & & & & 0 & 0 \\
\hline
\end{tabular}

Cusco; así como el número de capítulos cosechados y el número total de semillas llenas obtenidas por cada tipo de cruzamiento. Viabilidad y germinación de semillas.

De los 300 capítulos polinizados en Cusco se obtuvo semilla en cantidades muy pequeñas y con apariencia bastante débil. Cuando las semillas fueron sumergidas en un vaso con agua, todas flotaron, lo cual fue el indicador para concluir que el $100 \%$ de las semillas obtenidas en Cusco fueron vanas o vacías. En Ambo fueron polinizados un total de 1746 capítulos y mayoritariamente se cosecharon semillas vacías. Sin embargo también se obtuvieron 1181 semillas llenas (Tabla 2). Si asumimos que cada capítulo tuvo en promedio 15 flores femeninas y que todas las flores fueron polinizadas en el presente ensayo, entonces se podría inferir que el porcentaje de éxito de la polinización para obtener semillas llenas fue de $4.5 \%$ (1181 semillas llenas a partir de 26190 flores polinizadas).

Para realizar las pruebas de cromosomas en células metafásicas de una accesión octoploide y otra dodecaploide de yacón.

Polinizaciones.

El inicio de la floración fue diferente en las seis accesiones de yacón. Las accesiones V4 y V24 resultaron más precoces y florearon más temprano que las accesiones V11, V18, V28 y el Testigo. En términos generales la floración fue abundante en las seis accesiones, excepto en V11. Sin embargo, debido al elevado número de plantas que se sembraron por cada accesión, esto no representó un obstáculo para la realización de las polinizaciones. La Tabla 2 muestra el número de inflorescencias polinizadas por cada tipo germinación las 1181 semillas llenas fueron divididas en dos grupos. Para la prueba de germinación en placas Petri se emplearon 759 semillas (Tabla 4) y para la prueba de germinación en sustrato orgánico (turba y musgo) se emplearon 422 semillas (Tabla 5). El método de germinación en placas Petri no funcionó para ninguna de las 759 semillas a pesar que las placas Petri fueron evaluadas durante seis semanas (Tabla 4). En cambio, la germinación de semillas en sustrato orgánico (musgo y turba) funcionó muy bien, lográndose obtener 38.4\% de germinación (162 de las 422 semillas llenas lograron germinar). 

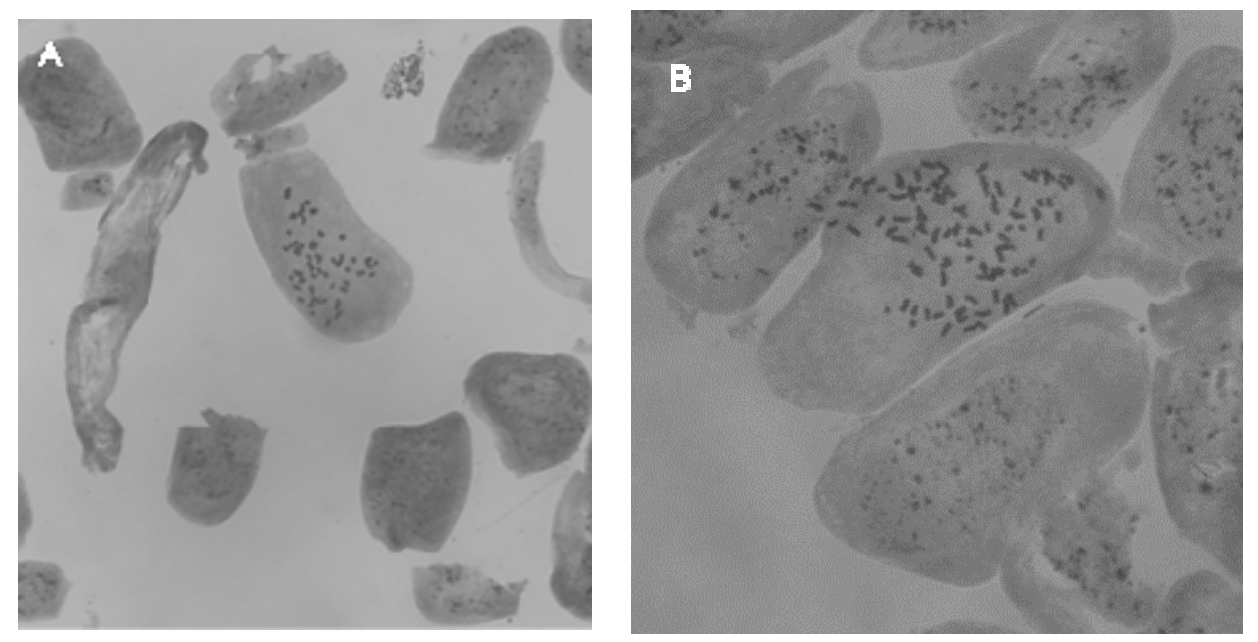

Figura 4. A: Célula metafásica de la accesión V11 (octoploide) con 58 cromosomas. B: Célula metafásica de la accesión V28 (dodecaploide) con 87 cromosomas.

El inicio de la germinación de las semillas se dio entre el sétimo y décimo día después de la siembra en el sustrato orgánico. Las semillas germinadas fueron mantenidas en el mismo sustrato por 30 días y luego fueron transferidas a macetas de 3.5 pulgadas. Finalmente, las plántulas fueron sembradas en campo definitivo en la comunidad de Rosapata (Ambo).

Los cruzamientos entre accesiones octoploides (V4, V11, V18 y V24) siempre produjeron semillas llenas. Sin embargo los cruzamientos entre accesiones dodecaploides (V28xT y TxV28) nunca produjeron semillas llenas ni viables (Tabla 2). Cuando las accesiones dodecaploides (V28 y T) actuaron como progenitores femeninos tampoco se obtuvieron semillas llenas ni viables, excepto para el cruzamiento TxV11 (aunque solo lograron germinar cuatro semillas). Sin embargo, cuando las accesiones dodecaploides actuaron como progenitores masculinos siempre se obtuvieron semillas llenas (Tabla 2) y viables (entre 17 y 47\% de viabilidad) (Tabla 5).

\section{Discusión.}

La producción de semillas llenas y viables pudo ser lograda en condiciones de Ambo pero no en Cusco. Aunque en Cusco se obtuvieron semillas, todas resultaron vanas o parcialmente llenas. Las razones de estas diferencias pueden ser varias, pero probablemente como en muchos otros cultivos estén asociadas a la fecha de siembra de los progenitores, a la fecha en que se realizaron las polinizaciones y a las características particulares de cada localidad (Fenwick \& George 1988; Thomsen, 1997). En Ambo se sembraron las plantas en el mes de junio de 2011 y se realizaron las polinizaciones entre los meses de marzo y abril de 2012. En Cusco en cambio la siembra y las polinizaciones se realizaron dos meses más tarde que en Ambo. Estas diferencias podrían ejercer un fuerte efecto en el desarrollo fisiológico de las semillas y afectar su posterior viabilidad. Es conocido también que la altitud y las condiciones de suelo y clima (temperatura y humedad) son factores que influyen en la calidad y cantidad de semillas producidas en diferentes especies de plantas (Cavers et al., 2000; Gutterman, 2000).

La germinación de semillas en varias especies de plantas no es posible sin un tratamiento previo de escarificación. Grau \& Slanis (1996; citados por Grau \& Rea, 1997) han reportado que la producción de semilla en $S$. macroscyphus (un pariente silvestre relacionado al yacón) es muy alta pero su germinación es impedida por la cubierta dura de las semillas. En la presente investigación se determinó que las semillas de yacón pudieron germinar únicamente cuando los frutos fueron escarificados. Según Seminario et al. (2003) el pericarpio de los aquenios es delgado y seco. Sin embargo, el pericarpio representaría de todas maneras una barrera lo suficientemente impermeable o resistente para permitir el ingreso de aire y humedad al interior del fruto e inducir así el desarrollo y emergencia de la radícula.

Las experiencias previas de producción de semillas de yacón a partir de polinizaciones controladas han dado generalmente cifras muy bajas. En Argentina no se obtuvieron semillas llenas a partir de cruzamientos realizados en un solo clon de yacón (Grau, 1993; citado por Grau \& Rea, 1997). En la presente investigación se logró obtener 1181 semillas llenas a partir de 1746 capítulos polinizados en condiciones de campo abierto en una comunidad de la serranía de Ambo. Si asumimos que cada capítulo tenía en 
Tabla 5. Resultados de la prueba de germinación utilizando sustrato orgánico (turba y musgo).

\begin{tabular}{|c|c|c|c|c|c|}
\hline $\begin{array}{l}\text { Progenitor } \\
\text { Femenino }\end{array}$ & $\begin{array}{l}\text { Progenitor } \\
\text { Masculino }\end{array}$ & $\begin{array}{l}\text { Nro. capítulos } \\
\text { procesados }\end{array}$ & Nro. semillas llenas & $\begin{array}{l}\text { Nro. semillas germinadas } \\
\text { y trasplantadas a campo }\end{array}$ & $\begin{array}{l}\text { Porcentaje de } \\
\text { germinación }\end{array}$ \\
\hline V4 & V11 & 40 & 19 & 5 & $26 \%$ \\
\hline V4 & V28 & 10 & 9 & 3 & $33 \%$ \\
\hline V11 & V4 & 48 & 81 & 27 & $33 \%$ \\
\hline V11 & V18 & 52 & 37 & 8 & $22 \%$ \\
\hline V11 & V24 & 54 & 38 & 17 & $45 \%$ \\
\hline V11 & V28 & 47 & 45 & 15 & $33 \%$ \\
\hline V11 & $\mathrm{T}$ & 34 & 23 & 4 & $17 \%$ \\
\hline V18 & V4 & 3 & 9 & 0 & $0 \%$ \\
\hline V24 & V4 & 56 & 9 & 5 & $56 \%$ \\
\hline V24 & V11 & 53 & 32 & 21 & $66 \%$ \\
\hline V24 & V18 & 71 & 40 & 20 & $50 \%$ \\
\hline V24 & V28 & 42 & 17 & 7 & $41 \%$ \\
\hline V24 & $\mathrm{T}$ & 47 & 15 & 7 & $47 \%$ \\
\hline V28 & $\mathrm{T}$ & 38 & 1 & 0 & $0 \%$ \\
\hline $\mathrm{T}$ & V11 & 14 & 19 & 4 & $21 \%$ \\
\hline V4 & Bulk & 4 & 11 & 6 & $55 \%$ \\
\hline V24 & Bulk & 12 & 17 & 13 & $76 \%$ \\
\hline Total & & 625 & 422 & 162 & $38 \%$ \\
\hline
\end{tabular}

promedio 15 flores femeninas y que todas las flores fueron polinizadas, entonces el porcentaje de éxito de la polinización para obtener semillas llenas fue de 4.5\%. El porcentaje de germinación de las semillas llenas fue $38.4 \%$ y todas las plántulas regeneradas alcanzaron un desarrollo vigorosos cuando fueron trasplantadas a campo. Este valor es significativamente mayor a lo reportado previamente por Mansilla et al. (2010) quienes obtuvieron una sola semilla viable a partir de 397 semillas cosechadas, lo que apenas representa una viabilidad de $0.25 \%$.

Chicata (1998) reportó una germinación de 29.1\% (357 de 1227) en semillas cosechadas a partir de un campo de polinización abierta en Cusco en el que se sembraron 67 accesiones de yacón del banco de germoplasma de la Universidad San Antonio Abad del Cusco. El porcentaje de germinación se elevó a 64.9\% cuando solo se consideraron las semillas llenas. Los resultados de Chicata (1998) guardan mayor concordancia con los resultados obtenidos en el presente estudio y contradicen los resultados de investigaciones previas en las que se sugiere que la producción de semilla de yacón es escasa, tiene poca viabilidad y poco vigor (Grau \& Rea, 1997; Meza, 1995; Mansilla et al., 2010). Considerando el porcentaje de germinación y el número de semillas llenas que se obtuvieron en la presente investigación se puede inferir que se necesitarían polinizar cuatro inflorescencias (exactamente 58 flores femeninas) para obtener una semilla viable de yacón. Esta cantidad es mucho menor a lo reportado previamente por Sugiura et al. (2007), quienes lograron obtener únicamente una semilla viable a partir de 382 inflorescencias polinizadas ( $0.26 \%$ de éxito).

La pérdida de viabilidad en las semillas de todas las especies vegetales está en función del tiempo y de las condiciones de almacenamiento, entre otros factores (Bernal-Lugo et al., 2000). En el presente estudio las semillas cosechadas de yacón fueron almacenadas por cinco meses a temperatura ambiente hasta el momento en que se realizaron las pruebas de germinación en sustrato orgánico. El tiempo y la temperatura de almacenamiento podrían haber disminuido la viabilidad de las semillas. Por lo tanto, aunque se obtuvo un porcentaje de germinación de $38.4 \%$ en las semillas llenas, es probable que este valor hubiese sido mayor si se hubiesen realizado los ensayos de germinación en un tiempo menor a cinco meses. Chicata (1998) logró una germinación de $64.9 \%$ en las semillas llenas, pero los ensayos de germinación los realizó dos meses después de haber cosechado las semillas.

Ishiki et al. (1997) han propuesto que el yacón es una especie alopoliploide originada por el cruzamiento de dos especies silvestres relacionadas, presumiblemente $S$. macroscyphus y $S$. riparius. Esta hipótesis es consistente con los niveles de ploidía y el número de cromosomas reportados por varios investigadores en diferentes accesiones de yacón, incluyendo los resultados obtenidos en la presente 
investigación. Según el modelo de Ishiki et al. (1997), los genotipos octoploides $(2 \mathrm{n}=6 \mathrm{~A}+2 \mathrm{~B}=58)$ producirían gametos balanceados $(n=3 A+1 B=29)$ y los dodecaploides $(2 \mathrm{n}=9 \mathrm{~A}+3 \mathrm{~B}=87)$ gametos desbalanceados con menores posibilidades de originar un embrión viable y un endospermo funcional en comparación a los gametos producidos por los octoploides. En la presente investigación se obtuvo semillas llenas y viables en los cruzamientos entre progenitores octoploides pero no se logró obtener semillas en los cruzamientos entre progenitores dodecaploides, lo cual resulta concordante con el modelo de Ishiki et al. (1997). Sin embargo, en los cruzamientos en donde el progenitor dodecaploide actuó como masculino y el octoploide como femenino se obtuvo semillas llenas y viables, un resultado que no encaja dentro del modelo. Una posible explicación es que las semillas obtenidas fueron el resultado de autopolinizaciones que ocurrieron antes de realizar las polinizaciones programadas con el genotipo dodecaploide. También podría haber sucedido que al momento de realizar las polinizaciones programadas, las flores femeninas habían sido polinizadas previamente por otras accesiones, las cuales deberían haber sido octoploides. Finalmente existe la posibilidad que las semillas (o una parte de ellas) se hayan formado por apomixis, un evento común en varias especies de la familia Asteracea que sin embargo no ha sido reportado en ninguna de las especies del género Smallanthus (Noyes, 2007). La caracterización citológica, morfológica y molecular de las plantas F1 podrá generar información adicional que ayude a entender mejor un componente de la biología reproductiva del yacón que aún es poco comprendido. Complementariamente, la evaluación de la capacidad de producción de semilla sexual en los híbridos F1 (en condiciones controladas) proporcionará información valiosa para continuar evaluando la hipótesis del origen alopoliploide del yacón y para determinar además si existe un mecanismo de androesterilidad como ha sido sugerido previamente por Mansilla et al. (2010).

\section{Conclusiones.}

La producción de semillas de yacón es influenciada por varios factores, entre los que podrían destacar el ambiente (localidad, temperatura, altitud, etc.), la fecha de siembra de los parentales, la fecha de las polinizaciones y la fecha de cosecha de los frutos. La germinación y viabilidad de las semillas llenas es relativamente alta (encima de 38\%), lo que demuestra que la reproducción sexual a través de polinizaciones controladas es factible de lograr. La población F1 generada en esta investigación permitirá diseñar estudios complementarios sobre la biología reproductiva y el origen alopoliploide del yacón, así como desarrollar estrategias de mejoramiento genético y eventualmente obtener variedades nuevas. Por último, la posibilidad de producir semillas de yacón plantea retos para la investigación de métodos alternativos de conservación y distribución de germoplasma de yacón.

\section{Agradecimientos.}

Al Instituto Nacional de Innovación Agraria (INIA) por proveer las accesiones de yacón que se usaron como parentales en las cruzas dialélicas. Al Ing. Miguel Valderrama, por su contribución en la capacitación de técnicas de cruzamientos en yacón y por proporcionar varias de las fotografías usadas en el presente manuscrito. Esta investigación pudo ser realizada con el financiamiento de FIDECOM (Fondo de Investigación y Desarrollo para la Competitividad) del Ministerio de la Producción del Perú y de la empresa Andean Roots SRL.

\section{Literatura citada.}

Araujo H. 1998. Determinación del método para el contaje del número cromosómico en nueve cultivares de tres especies tuberosas andinas. Tesis de Grado, Universidad Nacional de Cajamarca, Perú, 45 p.

Bernal-Lugo I., Camacho A. \& Carballo A. 2000. Effects of seed ageing on the enzymic antioxidant system of maize cultivars. En: Seed Biology, Advances and Applications. Black M, Bradford K \& Vásquez-Ramos J (Eds.). Proceedings of the Sixth International Workshop on Seeds, Mérida, México, 1999. Pp. 151-160.

Cavers P., Qaderi M., Manku R. \& Downs M. 2000. Intermittent germination: causes and ecological implications. En: Seed Biology, Advances and Applications. Black M, Bradford K \& Vásquez-Ramos J (Eds.). Proceedings of the Sixth International Workshop on Seeds, Mérida, México, 1999. Pp. 363-374.

Chicata Pacheco R. 1998. Viabilidad de la semilla botánica y comparación fenotípica de progenies y clones provenientes del germoplasma de yacón (Polymnia soncifolia). Tesis de Grado, Universidad Nacional San Antonio Abad del Cusco, Perú, 152 p.

Dafni A. 1992. A Pollinization ecology: a practical approach. New York: IRL, 250 p.

Fenwick K \& Raymond G. 1988. Principles of seed production. En: Encyclopedia of seed Production of World Crops. John Wiley \& Sons Ltd, England. 403 p.

Frias de Fernandez A., Caro M., Lozzia de Canelada M. \& Grau A. 2000. Estudio citológico del yacón (Smallanthus sonchifolius) y yacón del campo (Smallanthus macroscyphus). Lilloa. (Argentina). 40(1):115-125.

Genta S., Cabrera C., Habib N., Pons J., Manrique I. Grau A. \& Sánchez S. 2009. Yacon syrup: Beneficial effects on obesity and insulin resistance in humans. Clinical Nutrition. 28(2):182-187.

Geyer M., Manrique I., Degen L. \& Beglinger C. 2008. Effect of yacon (Smallanthus sonchifolius) on colonic transit time in healthy volunteers. Digestion. 78(1):3033.

Grau A. \& Rea J. 1997. Yacon, Smallanthus sonchifolius (Poepp. \& Endl.) H. Robinson. In: Hermann M. \& J Heller (eds.): Andean roots and tubers: Ahipa, arracacha, maca yacon. Promoting the conservation and use of 
underutilized and neglected crops. 21. Institute of Plant Genetics and Crop Plant Research. Gatersieben/International Plant Genetic Resources Institute. Rome, Italy, p. 199-242.

Gutterman Y. 2000. Genotipic and phenotypic germination survival strategies of ecotypes and annual plant species in the Negev desert of Israel. En: Seed Biology, Advances and Applications. Black M, Bradford K \& Vásquez-Ramos J (Eds.). Proceedings of the Sixth International Workshop on Seeds, Mérida, México, 1999, p. 389-399.

Hermann M., Freire I. \& Pazos C. 1999. Compositional diversity of the yacon storage root. In: Impact on a changing world: Program report 1997-98. International Potato Center (CIP), Lima (Perú), p. 425-432.

Ishiki K., Salgado Moreno V. \& Arellano J. 1997. Revision of chromosome number and karyotype of Yacon (Polymnia sonchifolia). Resúmenes del primer taller internacional sobre recursos fitogenéticos del noroeste argentino. INTA, Salta, Argentina.

Mansilla R., López C., Flores M. \& Espejo R. 2010. Estudios de la biología reproductiva en cinco accesiones de Smallanthus sonchifolius (Poepp. \& Endl.) Robinson. Ecología Aplicada (Perú). 9(2):167-175.

Meza G. 1995. Variedades nativas de Llacon (Polymnia sonchifolia Ker Gawler) en Cusco. UNSAAC-CICA, Kayra; CIP-COTESU.

Noyes RD. 2007. Apomixis in the Asteraceae: diamonds in the rough. Funtional Plant Science and Biotechnology. 1(2):207-222.

Salgado Moreno V. 1996. Evaluación y caracterización citogenética de 16 entradas de jícama (Polymnia sonchifolia Poep. \& Endl.) de Sudamérica. Tesis de Grado, Universidad Central del Ecuador, Ecuador.
Seminario J., Valderrama M. \& Manrique I. 2003. El yacón: Fundamentos para el aprovechamiento de un recurso promisorio. Lima (Perú). Centro Internacional de la Papa (CIP); Universidad Nacional de Cajamarca; Agencia Suiza para el Desarrollo y la Cooperación (COSUDE). $57 \mathrm{p}$.

Seminario J., Valderrama M., \& Romero J. 2004. Variabilidad morfológica y distribución geográfica del yacón, Smallanthus sonchifolius (Poepp. \& Endl.) H. Robinson, en el norte peruano. Arnaldoa (Perú). 11(1):139-160.

Soto Fernández R. 1998. Estudio de la biología floral del germoplasma regional de yacón. Tesis de Grado, Universidad Nacional de Cajamarca, Perú. 51 p.

Soto Torres J. 2012. Evaluación de la diversidad genética de colecciones de Smallanthus sonchifolius (Poepp. \& Endl.) "Yacón” del Perú. Tesis de Maestría, Universidad Nacional Agraria La Molina, Perú.

Sugiura M., Nakanishi T., Kameno T., Doi Y. \& Fujino M. 2007. A new yacon cultivar: Sarada Otome. Bulletin of the National Agricultural Research Center for Western Region (Japan). 6:1-13.

Thomsen K. 1997. The effect of harvest time and drying on dormancy and storability in beechnuts. En: Basic and Applied Aspects of Seed Biology. Ellis R, Black M, Murdoch A \& Hong T (Eds.). Proceedings of the Fifth International Workshop on Seeds, Reading, 1995. Kluwe Academic Publishers, London. 823 p.

Valentova K., Lebeda A., Dolezalova I., Jirovsky D., Simonovska B., Vovk I., Kosina P., Gasmanova N., Dziechciarkova M. \& Ulrichova J. 2006. The biological and chemical variability of yacon. Journal of Agricultural and Food Chemistry. 54(4):1347-1352.

1 Centro Internacional de la Papa (CIP). Av. La Molina 1895, La Molina, Lima, Perú. Correo electrónico: i.manrique@cgiar.org

2 Andean Roots SRL. Carretera Central Huánuco-Lima, km. 40, Ambo, Huánuco, Perú. Correo electrónico: a.valladolid@andeanroots.com.pe

${ }^{3}$ Universidad Nacional Agraria La Molina (UNALM). Av. La Molina s/n, La Molina, Lima, Perú. Correo electrónico: rblas@lamolina.edu.pe

${ }^{4}$ Universidad Nacional San Antonio Abad del Cusco (UNSAAC). Av. de la Cultura 733, Cusco, Perú. Correo electrónico: lizarragavl_daag@unsaac.edu.pe 